\title{
NON-OHMIC CONDUCTIVITY OF HIGH RESISTIVITY CdTeף
}

\author{
J. LuSakowski, J. Szczytko, K. Szadkowski \\ Institute of Experimental Physics, University of Warsaw \\ Hoża 69, 00-681 Warszawa, Poland \\ E. Kamińska, A. Piotrowska
}

Institute of Electron Technology, Al. Lotników 32/46, 02-668 Warszawa, Poland

\section{G. KarCZeWsini and T. WoJTowicz}

Institute of Physics, Polish Academy of Sciences

Al. Lotników 32/46, 02-668 Warszawa, Poland

\begin{abstract}
Conductivity measurements were carried out at room temperature on samples of nominally undoped Bridgman, bulk crystals and MBE-grown layers of CdTe. The samples were equipped with indium contacts which made it possible to determine the voltage distribution along the path of the current flow. The results show that for both types of CdTe almost all of the applied voltage drops in the vicinity of the positively biased contact. The resistance of the samples was shown not to depend on the distance between the pads. The results agree with predictions of model of current injection into semiconductors with deep traps.
\end{abstract}

PACS numbers: $72.80 .-\mathrm{r}$

\section{Introduction}

Results of theoretical and experimental investigation of the conductivity of semi-insulating GaAs [1-6] and CdTe [7, 8] allow to stress the important role of carrier injection into compensated semiconductors. In general, the injection, accompanied by generation and recombination processes which involve deep traps, leads to a nonuniform electric field distribution along the current. This is usually connected with the appearance of depletion zones, which cause an increase in the resistance with respect to that of an unperturbed bulk material. Semi-insulating GaAs is one of the most intensively investigated materials in this field. However,

"This work is partially supported by the State Committee for Scientific Research (Republic of Poland) grant No. 7 S 20102306. 
a rapidly growing interest in the properties of quasi two-dimensional II-VI structures demands a similar investigation to be carried out on highly resistive II-VI compounds. In particular, CdTe is particularly important due to its application as a substrate and also in solar cells and $\gamma$-ray detectors.

In this paper results of conductivity measurements on semi-insulating CdTe crystals are shown. An estimation of the electric field distribution in samples revealed a dominant role of the positively biased contact (the anode) on the resistance of the sample. A direct consequence of this fact is the lack of the resistance dependence on the length of a sample.

\section{Experiment and results}

Measurements were carried out at room temperature on samples of nominally undoped CdTe. Two types of materials were investigated: bulk crystals, grown by the modified Bridgman method, and $3 \mu \mathrm{m}$ thick MBE layers grown on a semi-insulating $\mathrm{GaAs}$ substrate. Each sample was supplied with seven indium dot contacts, arranged in a row. The dimension of the dots and the distance between the centres of two neighbouring dots was equal to $0.2 \mathrm{~mm}$ and $0.5 \mathrm{~mm}$, respectively. The electrical circuit consisted of a sample connected in series with a Keithley 220 current source. The outputs of the current source were connected with the outermost contacts ( $3 \mathrm{~mm}$ apart). The voltage drop was measured by a Keithley 617 electrometer by connecting one of its inputs to one of the outputs of the current source, while the other input was subsequently connected with other contacts. In this way current-voltage characteristics were obtained (Fig. 1) which allowed to determine the electric potential distribution (Fig. 2). The positive part of the characteristic in Fig. 1 measures voltage from the anode to a contact in the inner part of the sample, while the negative part shows the voltage drop near the cathode.

\section{Discussion}

For both materials the characteristics are nonlinear and nonsymmetric with respect to the reversal of the direction of the current. For the bulk crystal all the characteristics coincide for the positive direction of the current, and all but one for the negative direction. This can be understood by inspection of Fig. 2, which shows that a dominant part of the applied voltage drops in the vicinity of the anode. Thus, if one input of the electrometer is connected to the anode, it is relatively of no importance to which contact the other input is connected. On the other hand, for the negative part of the characteristics, an essential voltage drop with respect to the cathode is measured only when the other input reaches the anode region. Thus, moving from the cathode to the anode one observes a plateau which stretches over almost all the length of the sample, and then a sharp increase near the anode. The spacing between the contacts allows to determine the width of the regions of high voltage gradients to be not wider than $0.3 \mathrm{~mm}$.

The voltage gradients lead to maxima in the electric field distribution, which is thus highly nonlinear. The gradient of the potential is much larger at the anode 

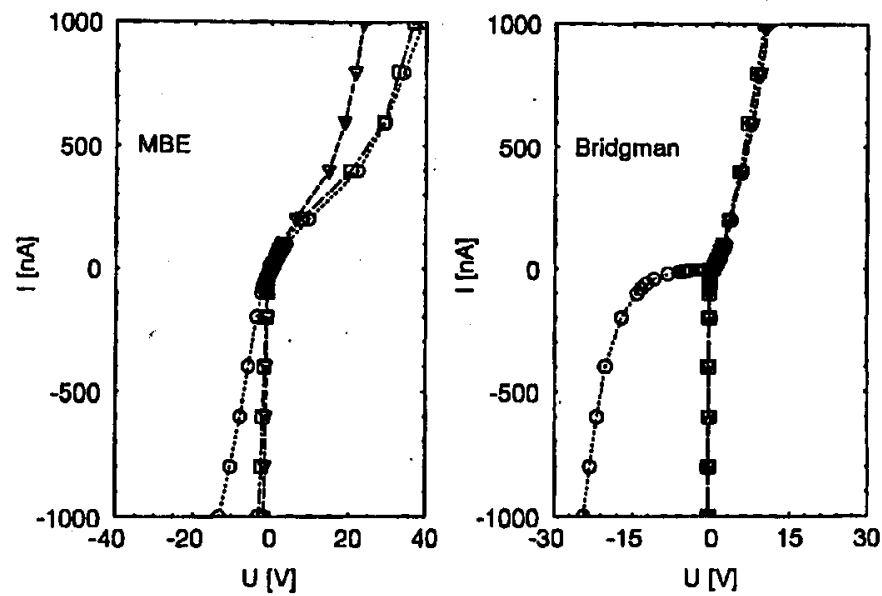

Fig. 1. Current-voltage characteristics of an MBE epitaxial layer (left) and a Bridgman bulk sample (right) of semi-insulating CdTe. The current $I$ was supplied by two outermost contacts ( $3 \mathrm{~mm}$ apart). The voltage drop $U$ was measured between one of these contacts and another one, which was situated at a distance $x$ varying from $0.5 \mathrm{~mm}$ to $3 \mathrm{~mm}$. The figure shows results for $x=0.5 \mathrm{~mm}$ (triangles), $1.5 \mathrm{~mm}$ (squares) and $3 \mathrm{~mm}$ (circles). Other characteristics were not plotted for the clarity of the figure. The part of each characteristics which corresponds to positive currents and voltages gives the potential difference between the anode and given contact, while the negative part gives a potential difference with respect to the cathode.
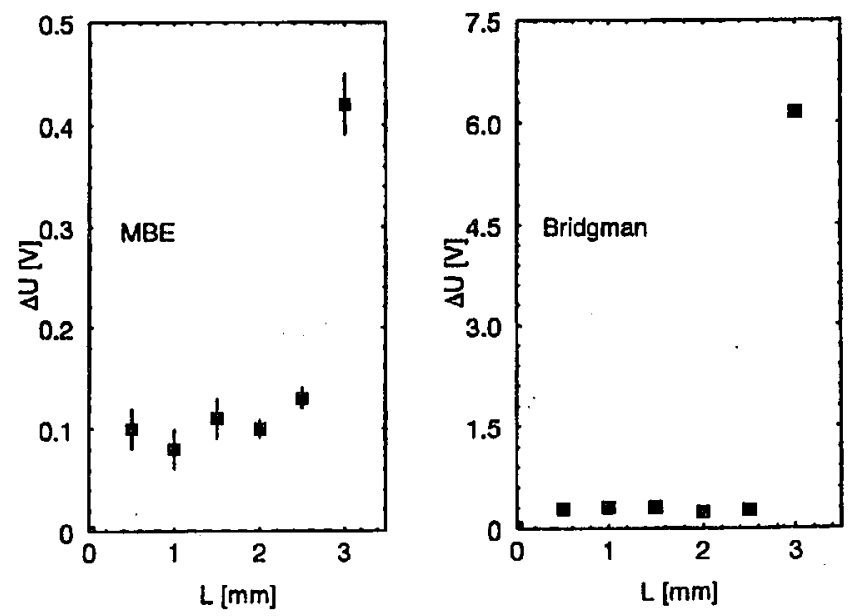

Fig. 2. The potential distribution along the current flow in an MBE epitaxial layer (left) and a Bridgman bulk sample (right) of semi-insulating CdTe, obtained on the base of Fig. 1. The cathode is situated at $L=0 \mathrm{~mm}$ and the anode at $L=3 \mathrm{~mm}$, while the current is $10 \mathrm{nA}$. In the case of the bulk sample, error bars are smaller than the point marks. 
than at the cathode. This difference is smaller for the MBE crystal than for the bulk one and decreases further for the MBE sample when the current grows. Since the electric field profile strongly depends on characteristics of the contacts, one can argue that the data in Fig. 1 and Fig. 2 show a direct relation between the quality of the material and the quality of the contacts. Smaller gradients for the cese of the MBE sample indicate that indium contacts to this material are "more chmic" than in the case of bulk samples.

A large drop of the potential near the positively biased contact allows to argue that the resistance of a sample should not depend on its length, provided the sample is longer than the extent of the anode region. This was in fact observed by measuring the voltage drop between two outputs of the current source, which were connected to different pairs of contacts.

All the observed features of the potential distribution are known to be typical phenomena resulting from current injection into semiconductors with deep traps. In particular, these problems were analysed by Manifacier and Henish [4] by means of a set of linearized transport equations. An important point in their approach is treating contacts as boundary conditions of the problem and describing them by an injection ratio [2], which becomes an adjustable parameter of a solution. These authors applied their analysis to semi-insulating GaAs and showed that injection of minority carriers into this material may cause the resistance of a sample to be independent of its length. This is exactly the result obtained in this paper. Thus, application of the model [4] to our data may result in getting some practical information on injection through indium contacts into CdTe.

\section{References}

[1] W. van Roosbroeck, Phys. Rev. 123, 474 (1961).

[2] J.-C. Manifacier, H.K. Henish, Phys. Rev. B 17, 2640 (1978).

[3] J.-C. Manifacier, H.K. Henish, Phys. Rev. B 17, 2648 (1978).

[4] J.-C. Manifacier, H.K. Henish, J. Appl. Phys. 52, 5195 (1981).

[5] N. Derhacobian, N.M. Heagel, Phys. Rev. B 44, 12754 (1991).

[6] N. Derhacobian, J.H. Madok, N.M. Hegel, J. Appl. Phys. 72, 2294 (1992).

[7] A.M. Mancini, C. Manfredotti, C. de Blasi, G. Miccocci, A. Tepre, Rev. Phys. Appl. 12, 255 (1977).

[8] R. Graft, J. Dinan, U. Lee, T. Fischer, J. Ramsey, T. Golding, H. Wilson, J. Vac. Sci. Technol. A 7, 377 (1988). 